\title{
Proportional Fair Scheduling: Analytical Insight under Rayleigh Fading Environment
}

\author{
Erwu Liu and Kin K. Leung \\ Department of Electrical and Electronic Engineering \\ Imperial College \\ London, United Kingdom \\ \{erwu.liu,kin.leung@imperial.ac.uk\}
}

\begin{abstract}
This paper provides analytical expressions to evaluate the performance of a random access wireless network in terms of user throughput and network throughput, subject to the constraint of proportional fairness amongst users. The proportional fair scheduling (PFS) algorithm is considered an attractive bandwidth allocation criterion in wireless networks for supporting high resource utilization while maintaining good fairness among network flows. The most challenge of a PFS problem is the lack of analytic expression. Though the PFS algorithm has been a research focus for some time, the results are mainly obtained from computer simulations. It is known that a PFS problem is NP-hard and, until recently, there are very few papers which give analytic insights into the PFS algorithm. Typically, existing works use simplified form of the PF preference metric and assume simple linear model, or the given analytic expression is valid only for very limited cases. In this research, we give analytical results of the PFS algorithm by providing closed-form expressions for the throughput in Rayleigh fading networks. We use Gaussian approximation method to model the feasible data rate in Rayleigh fading environments. Results obtained from the simulation and numerical analysis verifies the high accuracy of the closed-form expressions given in the paper. In particular, the analytic expressions given here will provide great help for the system design of a PFS-enable network, not only in that it is obtained from more realistic rate model, but also it applies to various kinds of network scenarios.
\end{abstract}

Keywords-proportional fair scheduling, gaussian approximation, rayleigh fading

\section{INTRODUCTION}

Scheduling policy plays an important role on system performances such as throughput, delay, jitter, fairness and loss rate [1]. Different from wired cases, scheduling in wireless networks need to consider the unique characteristics of timevarying and location-dependent channel status. Among various related researches on scheduling, the proportional fair scheduling (PFS) algorithm has been widely conceived as an attractive solution since it provides a good compromise between the maximum throughput and user fairness by exploiting multi-user diversity and game-theoretic equilibrium in fading wireless environment [2]. Due to its low implementation complexity and good performance, the PF scheduler has received much attention for some time [3] [4]. It is known that a PF problem is NP-hard [5] [6], and in most researches [7] [8], the results are obtained from computer simulations. Holtzman [9] and Kushner [10] conducted the asymptotic analysis of the PFS algorithm. However, none of the researches aforementioned provides analytic expression of the throughput of PFS. Recently, [4] and [11] analyzed the PFS algorithm with the objective of obtaining an analytic expression for the throughput. Using the ratio of the instantaneous signal to interference-plus-noise ratio (SINR) to the average SINR as the preference metric instead of the original PF metric of the ratio of the feasible rate to the average rate (or throughput), by assuming the SINR of user follows exponential distribution, [11] obtained an analytic expression for the user throughput of PFS. While in [4], the closed-form expression obtained is valid only for networks where there are large numbers of user. Though the analytic results given in [11] and [4] are obtained either for a simplified form of the original PFS preference metric or for large user number case, so far as we know, the formulae presented in [11] and [4] are the only two closed-form expressions available for the throughput of the PFS algorithm. In this paper, we analyze the PF scheduler under more realistic network scenarios and derive accurate closed-form expression for both network throughput and user throughput without the limitation of [4] and [11].

The rest of the paper is organized as follows. In Section II, we recapitulate the principles of the PFS algorithm and the Gaussian approximation to channel throughput. Then we obtain analytical results in terms of mean and standard deviation of the user throughput and the network throughput of PFS in an environment with Rayleigh fading in Section III. In Section IV, simulations are conducted to validate the analytic expression for various scenarios. In particular, we show that our closedform formulae for the throughput of PFS provide highly accurate estimates of simulation results. We give a brief conclusion in Section V.

\section{PROPORTIONAL FAIR SCHEDULING AND GAUSSIAN APPROXIMATION}

In this section, we first described the principles of the PFS algorithm, then the Gaussian approximation [12] [13] to the instantaneous data rate is outlined.

\section{A. Proportional Fair Scheduling Criteria}

Consider a single-cell system shown in Fig. 1, $N$ mobile users (denoted as users $m_{l}, m_{2}, \ldots$, and $m_{N}$ ) are randomly located within the cell served by a base station (BS). 


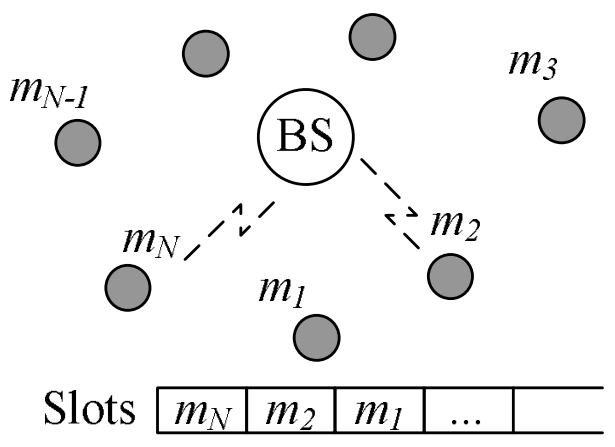

Figure 1. Single-cell network

Consider the problem where these $N$ users wishing to transmit data from the base station to $N$ destinations, and the rates of transmission are randomly varying. Time is divided into small scheduling intervals, called slots. Until further notice, in each slot only one user is chosen to transmit. In next scheduling slot, system will estimate the rates by estimating the SINR, by use of a pilot signal broadcasted periodically, with a very short delay. The selection of the user to schedule is based on a balance between the current possible rates and fairness. The proportional fair scheduling (PFS) algorithm [2] [4] [9] [10] performs this by comparing the ratio of the feasible rate for each user to its average throughput tracked by an exponential moving average, which is defined as the preference metric. The user with the maximum preference metric will be selected for transmission at the next scheduling slot. This is described mathematically as follows. The end of slot $n$ is called time $n$. In next time slot $n+1$, the instantaneous data rate of user $j$ will be $R_{j}[n+1]$. Its $k$-point moving average throughput up to time $n$ is $\overline{r_{j, k}}[n]$ denoted by, and the preference metric $\operatorname{by}_{M_{j ; k}[n+1]=R_{j}[n+1] / \overline{r_{j ; k}}[n]}$.

User $i=\arg \max _{j} M_{j ; k}[n+1]=\arg \max _{j} R_{j}[n+1] / \overline{r_{j ; k}}[n]$ is scheduled to transmit in next time slot $n+1$. From the definition of a $k$-point moving average, the moving average throughput user $j$ up to time $n+1$ of is updated by

$\overline{r_{j ; k}}[n+1]=\left(1-\frac{1}{k}\right) \overline{r_{j ; k}}[n]+I_{j}[n+1] \times \frac{R_{j}[n+1]}{k}$

where $I_{j}[\mathrm{n}+1]$ is the indicator function of the event that user $j$ is scheduled to transmit in time slot $n+1$.

$I_{j}[n+1]=\left\{\begin{array}{l}1, \text { user } j \text { scheduled in slot } n+1 \\ 0, \text { else }\end{array}\right.$

Obviously, for a time-varying fading environment, when the user number is large, there will always be high probability that some users are in the good channel status. On the other hand, the PFS algorithm provides some sense of fairness in that users frequently in the bad channel status have low throughput which in turn tends to increase their probability of being scheduled. It is the logarithm utility maximization characteristics, the multi-user diversity gains and the possibility to schedule bad-channel-condition users that make the PF scheduler superior to the traditional ones such as round-robin (RR) and opportunistic scheduler.
Though the PFS algorithm described by (1) and (2) has fairly low implementation complexity, it is in fact NP-hard to obtain its analytic solution [5].

\section{B. Gaussian Approximation Method}

Though it is a common practice to assume that the instantaneous data rate of a user is linearly proportional to its SINR [11] [14], this simple linear model is unrealistic and valid only for very small SINR. Various works [12] [13] have shown that the instantaneous data rate of a user in a Rayleigh fading, multiple-input-multiple-output (MIMO) wireless system could be more accurately approximated by a Gaussian distribution. Simulation results presented in [13] verified that the Gaussian approximation itself is surprisingly accurate for virtually all values of $t, r$, where $t, r$ are the number of transmit antennas and receive antennas of each user, respectively. Refer to [13], for single-input-single-output (SISO) case $(t=r=1)$, we can formally describe the Gaussian approximation method as follows: The instantaneous data rate $R$ (in $\mathrm{bps} / \mathrm{Hz}$ ) for a user is Gaussian distribution,

$$
\begin{array}{r}
E[R]=\int_{0}^{\infty} \log (1+\operatorname{SINR} \times \lambda) \times e^{-\lambda} d \lambda \\
\sigma_{R}^{2}=\int_{0}^{\infty}(\log (1+\operatorname{SINR} \times \lambda))^{2} \times e^{-\lambda} d \lambda \\
-\left(\int_{0}^{\infty} \log (1+\operatorname{SINR} \times \lambda) \times e^{-\lambda} d \lambda\right)^{2}
\end{array}
$$

where $\operatorname{SINR}$ is the signal to interference-plus-noise ratio, $E[R]$ and $\sigma_{R}$ are the mean value and the standard deviation of $R$, respectively.

\section{PfS: ClOSE-Form EXPRESSION}

From (1), the expect value of the $k$-point moving average throughput of user $j$ up to time $n+1$ is

$$
\begin{aligned}
E\left[\overline{r_{j ; k}}[n+1]\right] & =E\left[(1-1 / k) \overline{r_{j ; k}}[n]+I_{j}[n+1] \times R_{j}[n+1] / k\right] \\
& =\left(1-\frac{1}{k}\right) E\left[\overline{r_{j ; k}}[n]\right]+\frac{E\left[I_{j}[n+1] R_{j}[n+1]\right]}{k}
\end{aligned}
$$

And from (2),

$$
\begin{aligned}
E\left[I_{j}[n+1] R_{j}[n+1]\right] & =E\left[1 \times R_{j}[n+1] \mid I_{j}[n+1]=1\right] \\
& \times \operatorname{Pr}\left(I_{j}[n+1]=1\right) \\
& +E\left[0 \times R_{j}[n+1] \mid I_{j}[n+1]=0\right] \\
& \times \operatorname{Pr}\left(I_{j}[n+1]=0\right) \\
& =E\left[R_{j}[n+1] \mid I_{j}[n+1]=1\right] \\
& \times \operatorname{Pr}\left(I_{j}[n+1]=1\right)
\end{aligned}
$$

where $\operatorname{Pr}\left(I_{j}[\mathrm{n}+1]=1\right)$ is the average probability that user $j$ will be scheduled in time slot $n+1$.

Assuming wide-sense stationary $\overline{r_{j}}$ and applying Bayes's theorem, we can write (5) as 


$$
\begin{aligned}
E\left[\overline{r_{j ; k}}[n]\right] & =E\left[I_{j}[n+1] R_{j}[n+1]\right] \\
& =\operatorname{Pr}\left(I_{j}[n+1]=1\right) \times \int_{-\infty}^{\infty} x f_{R_{j}}\left(x \mid I_{j}[n+1]=1\right) d x \\
& =\int_{-\infty}^{\infty} x f_{R_{j}}(x) \operatorname{Pr}\left(I_{j}[n+1]=1 \mid R_{j}[n+1]=x\right) d x
\end{aligned}
$$

Under the PFS criteria presented in Section II,

$\operatorname{Pr}\left(I_{j}[n+1]=1 \mid R_{j}[n+1]=x\right)=\operatorname{Pr}\left(\forall i \neq j, \frac{R_{i}[n+1]}{\overline{r_{i ; k}}[n]}<\frac{x}{\overline{r_{j ; k}}[n]}\right)$

where $\overline{r_{j ; k}}[n]$ is the $k$-point moving average throughput of user $j$ up to time $n$.

For statistically independent $R_{j}$, (8) can be written in the form

$\operatorname{Pr}\left(I_{j}[n+1]=1 \mid R_{j}[n+1]=x\right)=\prod_{i=1, i \neq j}^{N} F_{R_{i}}\left(\frac{x}{\overline{r_{j ; k}}[n]} \overline{r_{i ; k}}[n]\right)$

By use of (1) when $k \rightarrow \infty$, we have

$$
\begin{aligned}
\lim _{k \rightarrow \infty} \frac{\overline{r_{i ; k}}[n]}{r_{j ; k}[n]} & =\lim _{k \rightarrow \infty} \frac{\overline{r_{i, k}}[n-1]}{r_{j ; k}[n-1]}=\ldots=\lim _{k \rightarrow \infty} \frac{\overline{r_{i ; k}}[1]}{\overline{r_{j ; k}}[1]} \\
& =\lim _{k \rightarrow \infty} \frac{\sum_{m=1}^{n} \overline{r_{i ; k}}[m]}{\sum_{m=1}^{n} \overline{r_{j ; k}}[m]}
\end{aligned}
$$

Hence,

$$
\begin{aligned}
\lim _{n \rightarrow \infty}\left\{\lim _{k \rightarrow \infty} \frac{\overline{r_{i ; k}}[n]}{\overline{r_{j ; k}}[n]}\right\} & ==\lim _{k \rightarrow \infty}\left\{\lim _{n \rightarrow \infty} \frac{\sum_{m=1}^{n} \overline{r_{i ; k}}[m]}{\sum_{m=1}^{n} \overline{r_{j ; k}}[m]}\right\} \\
& \left.=\lim _{k \rightarrow \infty} \frac{A\left[\overline{r_{i ; k}}\right]}{A\left[\overline{r_{j ; k}}\right.}\right]
\end{aligned}
$$

where $A[\cdot]$ denotes the time average.

For wide-sense stationary $\overline{r_{j ; k}}$, we further assume it is firstorder ergodic, then

$\lim _{n \rightarrow \infty}\left\{\lim _{k \rightarrow \infty} \frac{\overline{r_{i ; k}}[n]}{\overline{r_{j ; k}}[n]}\right\}=\lim _{k \rightarrow \infty} \frac{E\left[\overline{r_{i ; k}}\right]}{E\left[\overline{r_{j ; k}}\right]}=\frac{E\left[\overline{r_{i}}\right]}{E\left[\overline{r_{j}}\right]}$

Therefore, for large $n, k$, (9) can be written as

$$
\begin{aligned}
\operatorname{Pr}\left(I_{j}[n+1]=1 \mid R_{j}[n+1]=x\right) & =\prod_{i=1, i \neq j}^{N} F_{R_{i}}\left(\frac{x}{\overline{r_{j ; k}}[n]} \overline{r_{i ; k}}[n]\right) \\
& \approx \prod_{i=1, i \neq j}^{N} F_{R_{i}}\left(\frac{E\left[\overline{r_{i}}\right]}{E\left[\frac{\bar{r}_{j}}{j}\right]} x\right)
\end{aligned}
$$

On substitution of (13) into (7), we obtain

$$
E\left[\overline{r_{j}}\right] \approx \int_{-\infty}^{\infty} x f_{R_{j}}(x) \prod_{i=1, i \neq j}^{N} F_{R_{i}}\left(\frac{E\left[\bar{r}_{i}\right]}{E\left[\overline{r_{j}}\right]} x\right) d x
$$

Assuming $R_{j}$ is Gaussian as described in Section II,

$$
\begin{aligned}
E\left[\overline{r_{j}}\right] & =\int_{-\infty}^{\infty} x f_{R_{j}}(x) \prod_{i=1, i \neq j}^{N} F_{R_{i}}\left(E\left[\overline{r_{i}}\right] \times x / E\left[\overline{r_{j}}\right]\right) d x \\
& =\int_{-\infty}^{\infty}\left(y \sigma_{R_{j}}+E\left[R_{j}\right]\right) \times e^{-\frac{y^{2}}{2}} / \sqrt{2 \pi} \\
& \times \prod_{i=1, i \neq j}^{N} F_{R_{i}}\left(\left(y \sigma_{R_{j}}+E\left[R_{j}\right]\right) \times E\left[\overline{r_{i}}\right] / E\left[\overline{r_{j}}\right]\right) d y
\end{aligned}
$$

where $E\left[R_{j}\right], \sigma_{R j}$ are the expect value and standard deviation of $R_{j}$, respectively.

It is reasonable to guess that $E\left[\overline{r_{i}}\right] / E\left[\bar{r}_{j}\right]=E\left[R_{i}\right] / E\left[R_{j}\right]$ for PFS, we can then write

$\left.F_{R_{i}}\left(\frac{E\left[\overline{r_{i}}\right.}{E\left[\bar{r}_{j}\right.}\right]\left(y \sigma_{R_{j}}+E\left[R_{j}\right]\right)\right)=F_{(0,1)}\left(\frac{E\left[R_{i}\right] \sigma_{R_{j}}}{E\left[R_{j}\right] \sigma_{R_{i}}} y\right)$

where $F_{(0,1)}(\cdot)$ denotes zero mean, unit variance standard normal distribution function.

Finally, substituting (16) into (15) yields

$$
\begin{aligned}
E\left[\overline{r_{j}}\right] & =\int_{-\infty}^{\infty}\left(y \sigma_{R_{j}}+E\left[R_{j}\right]\right) / \sqrt{2 \pi} \times e^{-\frac{y^{2}}{2}} \\
& \times \prod_{i=1, i \neq j}^{N} F_{(0,1)}\left(\frac{E\left[R_{i}\right] \sigma_{R_{j}}}{E\left[R_{j}\right] \sigma_{R_{i}}} y\right) d y
\end{aligned}
$$

For the case where all users in the network have proportional mean and standard deviation, (17) reduces to

$$
\begin{aligned}
E\left[\bar{r}_{j}\right] & =\sigma_{R_{j}} \int_{-\infty}^{\infty} y \times \frac{e^{-\frac{y^{2}}{2}}}{\sqrt{2 \pi}}\left(F_{(0,1)}(y)\right)^{N-1} d y \\
& \left.+E\left[R_{j}\right]\right]_{0}^{1}\left(F_{(0,1)}(y)\right)^{N-1} d F_{(0,1)}(y) \\
& =\sigma_{R_{j}} \int_{-\infty}^{\infty} y \times \frac{e^{-\frac{y^{2}}{2}}}{\sqrt{2 \pi}}\left(F_{(0,1)}(y)\right)^{N-1} d y+\frac{E\left[R_{j}\right]}{N}
\end{aligned}
$$

where $N$ is the number of users in the network.

One can verify that equation (18) complies with our previous guess that $E\left[\overline{r_{i}}\right] / E\left[\overline{r_{j}}\right]=E\left[R_{i}\right] / E\left[R_{j}\right]$.

Now the closed-form expressions for the user throughput $E\left[\overline{r_{j}}\right]$ and network throughput $E\left[\overline{r_{P F S}}\right]$ of PFS are presented

$$
\left\{\begin{array}{l}
E\left[\overline{r_{j}}\right]=\frac{E\left[R_{j}\right]}{N}+\frac{\sigma_{R_{j}}}{N}\left[N \int_{-\infty}^{\infty} y \frac{e^{-\frac{y^{2}}{2}}}{\sqrt{2 \pi}}\left(F_{(0,1)}(y)\right)^{N-1} d y\right] \\
E\left[\overline{r_{P F S}}\right]=\frac{\sum_{j=1}^{N} E\left[R_{j}\right]}{N}+\frac{\sum_{j=1}^{N} \sigma_{R_{j}}}{N}\left[N \int_{-\infty}^{\infty} y \frac{e^{-\frac{y^{2}}{2}}}{\sqrt{2 \pi}}\left(F_{(0,1)}(y)\right)^{N-1} d y\right]
\end{array}\right.
$$

A few observations can be made from (19). First of all, as $E\left[R_{j}\right] / N$ is the mean throughput of user $j$ when using round robin (RR) scheduling, the second item in the right hand of (19) 
is in fact the improvement of user $j$ 's throughput when using PFS instead of RR scheduling. User $j$ 's throughput gain of PFS over RR scheduling is

$G_{P F S}(j)=1+\frac{\sigma_{R_{j}}}{E\left[R_{j}\right]}\left[N \int_{-\infty}^{\infty} z \frac{e^{-\frac{z^{2}}{2}}}{\sqrt{2 \pi}}\left(F_{(0,1)}(z)\right)^{N-1} d z\right]$

Obviously, PFS will benefit more in severe fading environments where $\sigma_{R j}$ is large. On the other hand, PFS can be viewed as RR scheduling in low fading environments where $\sigma_{R j}$ is very small. Interestingly, user $j$ 's throughput is solely determined by network user number $N$ and the characteristics of its own instantaneous data rate and thus not affected by other users in the network when using PFS.

By use of (3) and (4) in (20), a plot of $G_{P F S}$ vs. SINR for various $N$ is given in Fig. 2 (curves up to down are for $N=1000$, $500,200,70,20,5$ and 1, respectively). Fig. 2 shows $G_{P F S} \in(1.4,2.9)$ for a typical network scenario where $\operatorname{SINR\in }(6 \mathrm{~dB}, 28 \mathrm{~dB})$ and $N \in(20,200)$.

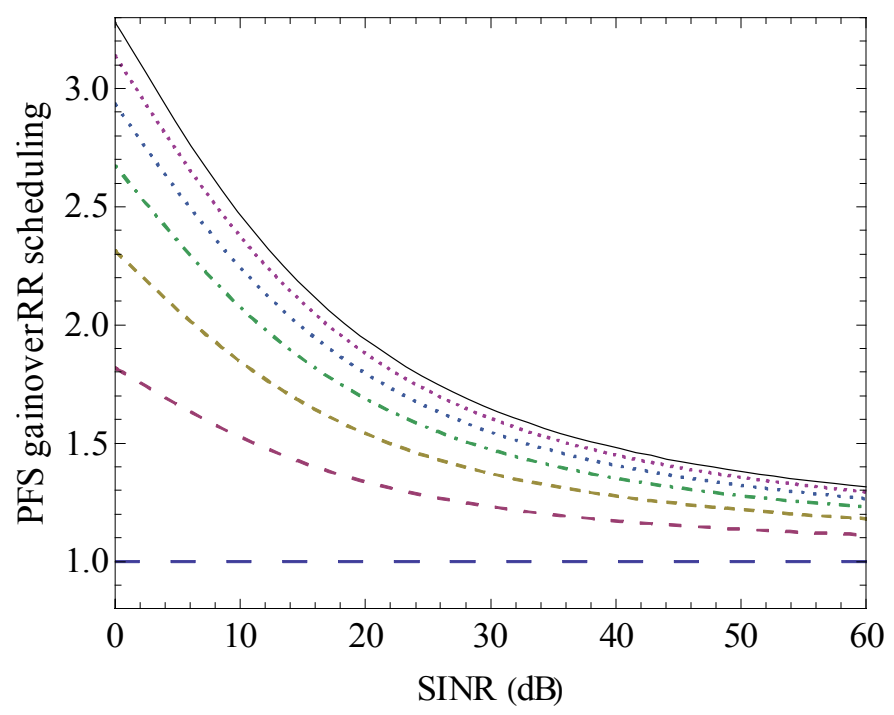

Figure 2. PFS gain vs. SINR for different number of users

Fig. 2 indicates the PFS gain $G_{P F S}$ (i.e., the multi-user diversity gain in [11]) is dependent on both user number $N$ and SINR while in [11] it is solely dependent on the user number $N$. In fact, for a good-channel-condition user (i.e., with large SINR), it will not benefit much from PFS as the PFS principle requires that it should provide compensation for the badchannel-condition users. Obviously, our analytic expression is more realistic than [11].

A comparison of the PFS gains between our analytic expression and the one provided in [11] is depicted in Fig. 3 for different $N$. A typical value of $\operatorname{SINR}=10 \mathrm{~dB}$ is assumed in the comparison. As shown in Fig. 3, [11] (the dashed line in the plot) unrealistically provides an over-optimistic estimate of the PFS gain.

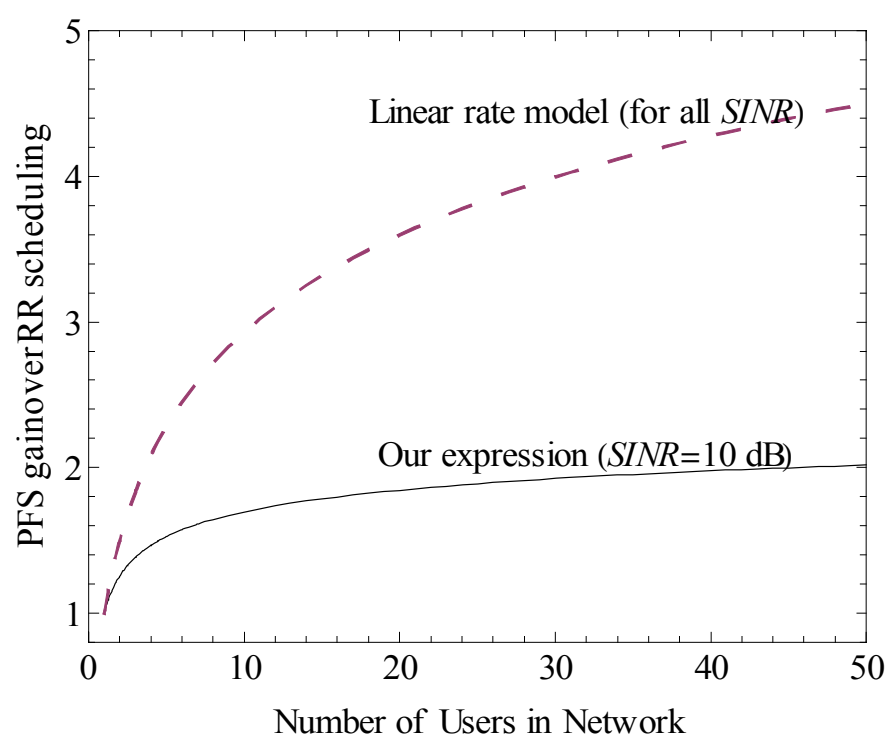

Figure 3. PFS gain plotted from (20) (the solid line) and [11] (the dashed one)

\section{VALIDITY OF THE ANALYTIC EXPRESSION BY COMPARISON WITH SIMULATION RESULTS}

The analytic expressions for the throughput of PFS are now investigated by comparing numerical and simulation results for various network scenarios. The instantaneous data rates of different users are statically independent. Initial moving average throughputs of users are randomized in the simulations. System parameters are: $20 \mathrm{MHz}$ bandwidth, $k=500$. For simplicity, we use the notation $n_{j}[\mu, \sigma]$ to indicate the instantaneous data rate of user $j$ has mean value $\mu$ and $\sigma$ (in Mbps), respectively.

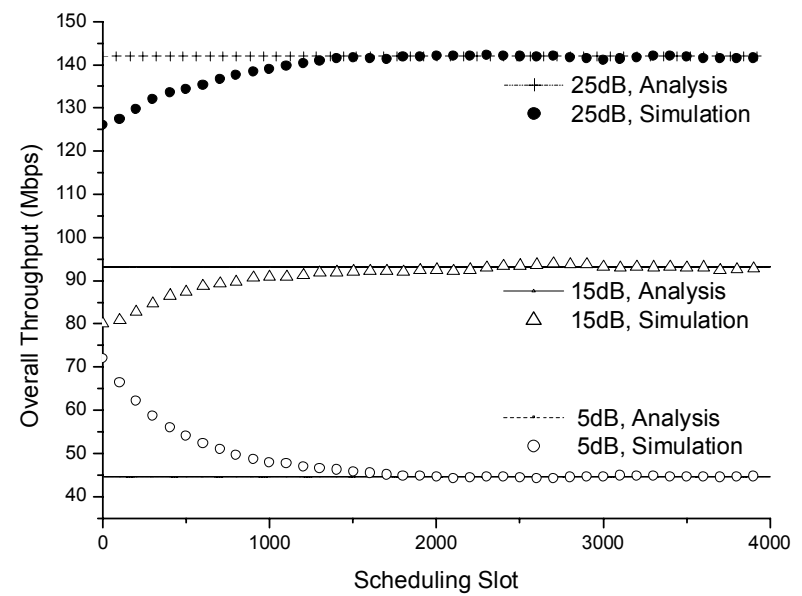

Figure 4. Accuracy of the analytic expression for overall throughput (Scenario I)

\section{A. Scenario I:}

The data rates for all users are i.i.d Gaussian distributions. This is the scenario that some researches [11] [14] uses to analyze the PFS problem. 
We plot in Fig. 4 and Fig. 5 the throughput of a 10-user network for some typical values of SINR.

Both figures show that the average throughputs from the simulations eventually converge to those determined by analytic expression (19).

To further verify the validity of our formulae, we plot as a reference in Fig.6 the normalized throughput $(\mathrm{bps} / \mathrm{Hz})$ vs. number of network users for various values of SINR.

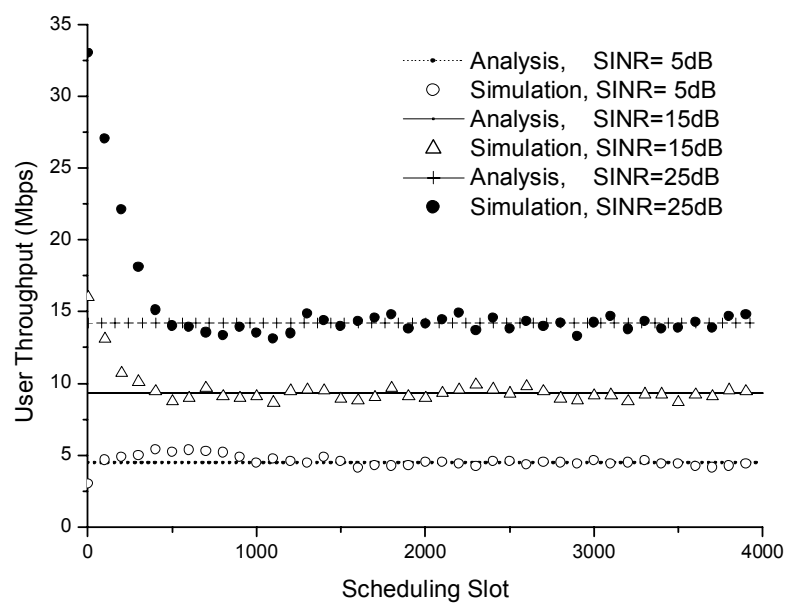

Figure 5. Accuracy of the analytic expression for user throughput (Scenario I)

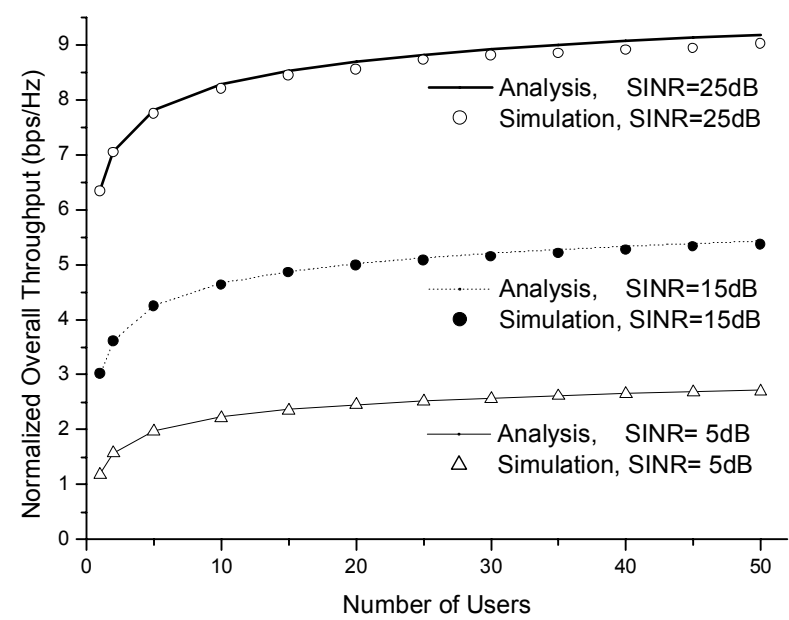

Figure 6. Accuracy of the analytic expression for different user number (Scenario I)

\section{B. Scenario II:}

User $j$ has randomized data rate mean $E\left[R_{j}\right]$ and proportional standard deviation $\sigma_{R j}$. In fact, scenario I is a special case of scenario II.

The feasible data rates (in Mbps) of users are randomized as:

$n_{1}[80,40] \quad n_{2}[40,20] \quad n_{3}[26,13] \quad n_{4}[80,40] \quad n_{5}[35,17.5]$ $n_{6}[28,14] \quad n_{7}[10,5] \quad n_{8}[38,19] \quad n_{9}[18,9] \quad n_{10}[45,22.5]$

where we assume the proportional factor is 0.5 .

Once again, Fig. 7 justifies the accuracy of equations (19)

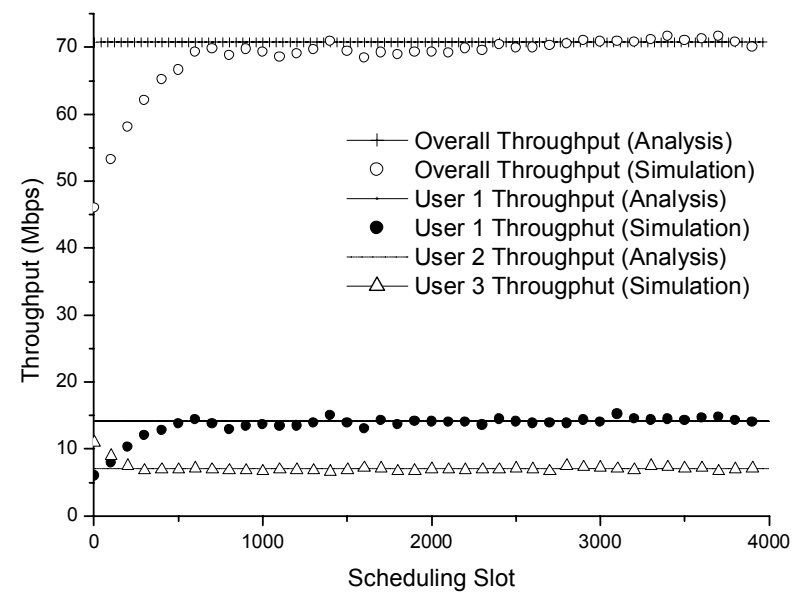

Figure 7. Accuracy of the analytic expression for overall/user throughput (Scenario II)

As expected, the simulation results in scenario I and II indicate a high accuracy of our closed-form formulae for PFS since the analytic expressions are obtained when assuming a proportional relationship between mean and standard deviation of moving average throughput.

Now we investigate the validity of the analytic expression under more realistic condition.

\section{Scenario III:}

User $j$ has randomized data rate mean $E\left[R_{j}\right]$ and standard deviation $\sigma_{R j}$ expressed by equations (3) and (4). Here we do not require proportional relationship between the mean and standard deviation of throughput.

The feasible data rates (in Mbps) of users are randomized as:

$n_{1}[43.3,18.9] \quad n_{2}[87.3,24.0] \quad n_{3}[24.1,13.7] \quad n_{4}[34.9,16.9] \quad n_{5}[129,25.3]$

$\begin{array}{llllll}n_{6}[91.8,24.2] & n_{7}[70.6,22.7] & n_{8}[37.4,17.6] & n_{9}[150,25.5] & n_{10}[27.7,14.9]\end{array}$

$n_{11}[45.9,19.4] \quad n_{12}[78.0,23.4] \quad n_{13}[34.2,16.8] \quad n_{14}[102,24.6] \quad n_{15}[86.9,23.9]$

$\begin{array}{llllll}n_{16}[49.9,20.1] & n_{17}[25.5,14.2] & n_{18}[32.5,16.3] & n_{19}[118,25.1] & n_{20}[79.4,23.5]\end{array}$

$n_{21}[141,25.4] \quad n_{22}[27.7,14.9] \quad n_{22}[27.7,14.9] \quad n_{24}[46.3,19.5] \quad n_{25}[37.8,17.6]$

$n_{26}[86.0,23.9] \quad n_{27}[70.6,22.7] \quad n_{28}[77.2,23.3] \quad n_{29}[127,25.2] \quad n_{30}[108,24.8]$

$n_{31}[86.0,23.9] \quad n_{32}[60.0,21.6] \quad n_{33}[133,25.3] \quad n_{34}[27.7,14.9] \quad n_{35}[86.0,23.9]$

$n_{36}[60.0,21.6] \quad n_{37}[47.1,19.6] \quad n_{38}[90.5,24.1] \quad n_{39}[158.9,25.5] \quad n_{40}[182,25.6]$

$n_{41}[64.2,22.1] \quad n_{42}[36.7,17.4] \quad n_{43}[34.2,16.8] \quad n_{44}[34.2,16.8] \quad n_{45}[42.1,18.6]$

$n_{46}[88.2,24.0] \quad n_{47}[65.5,22.2] \quad n_{48}[90.5,24.1] \quad n_{49}[71.9,22.9] \quad n_{50}[91.8,24.2]$

Clearly, the given user pattern reflects the real network scenario where different users may experience channel characteristics of great difference.

For a network with the user pattern above, the validity of (19) are illustrated in Fig. 8 and Fig. 9 in terms of normalized overall throughput and user throughput (bps/Hz). It is encouraging from both figures that our analytic expressions 
provide high accuracy even in scenario III which exhibits Rayleigh fading characteristics of a real wireless environment.

The overall conclusion from these experiments is that the closed-form expressions presented in Section III through analytic insights can be used with confidence to evaluate the performance of the PFS algorithm in various Rayleigh fading environments. The analytical results given in the paper will greatly facilitate the system design of a PFS-enable wireless network.

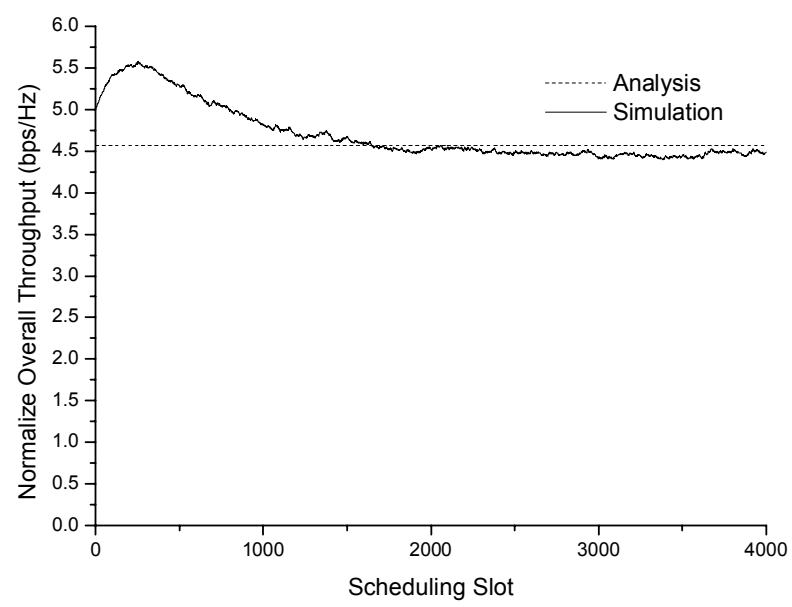

Figure 8. Accuracy of the analytic expression for overall throughput (Scenario III)

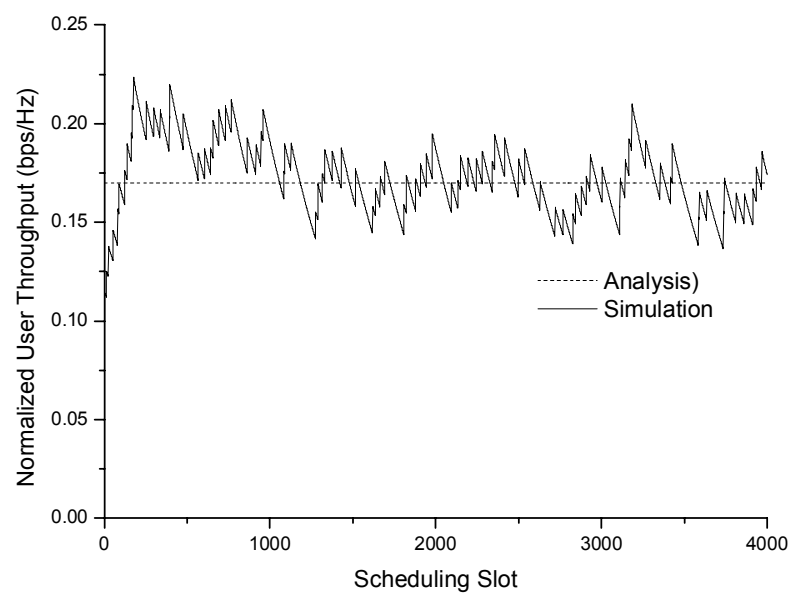

Figure 9. Accuracy of the analytic expression for user throughput (Scenario III)

\section{CONCLUSIONS}

In this paper, we develop analytic solution for the throughput of proportional fair scheduling in multi-user Rayleigh fading systems. Comparisons with simulation results have validated the analytic expressions presented in Section III.
By using Gaussian approximation to model instantaneous user data rate, the proposed closed-form expressions are more realistic than the existing one [11]. The intuitive yet formal formulas have the great practical and theoretical interests of being mathematically graceful and simple. Our theoretical results and findings provide guideline and analytical support on system design, simulation-based modeling and performance analysis of the PFS algorithm in the context of cross-layer design.

\section{ACKNOWLEDGMENT}

This research was financially supported by grants from EU IST FP6 MEMBRANE project under the contract 027310.

\section{REFERENCES}

[1] A. K. Parekh and R. G. Gallager, "A generalized processor sharing approach to flow control in integrated services networks: The singlenode case," IEEE/ACM Trans. Netw., vol. 1, no. 3, pp. 344-357, June 1993.

[2] F. Kelly, "Charging and Rate Control for Elastic Traffic", Eur. Trans. on Telecommun., February 1997, pp. 33-37.

[3] P. Viswanath, D. N. C. Tse, and R. Laroia, "Opportunistic beamforming using dumb antennas," IEEE Trans. Inf. Theory., vol. 48, no. 6, pp. 1277-1294, June 2002.

[4] G. Caire, R. Muller and R. Knopp, "Hard Fairness Versus Proportional Fairness in Wireless Communications: The Single-Cell Case," IEEE Trans. Inf. Theory., vol. 53, no. 4, pp. 1366-1385, April 2007.

[5] T. Bu, L. Li and R. Ramjee, "Generalized Proportional Fair Scheduling in Third Generation Wireless Data Networks," in Proc. INFOCOM, Barcelona, April 2006, pp. 1-12.

[6] T. D. Nguye and Y. Han, "A Proportional Fairness Algorithm with QoS Provision in Downlink OFDMA Systems," IEEE Commun Lett., vol. 10, no. 11, November 2006.

[7] H. Seo and B. G. Lee, "A Proportional-Fair Power Allocation Scheme for Fair and Efficient Multiuser OFDM Systems," in Proc. IEEE GLOBECOM, Dallas TX, 2004, pp. 3737-3741.

[8] Z. Han, Z. Ji and K. J. Ray Liu, "Fair Multiuser Channel Allocation for OFDMA Networks Using Nash Bargaining Solutions and Coalitions," IEEE Trans. Commun., vol. 53, no. 8, pp. 1366-1376, August 2005.

[9] J. M. Holtzman, "Asymptotic analysis of proportional fair algorithm," in Proc. IEEE PIMRC, San Diego, CA, 2001, pp. 33-37.

[10] H. J. Kushner and P. A. Whiting, "Asymptotic Properties of Proportional-Fair Sharing Algorithms: Extensions of the Algorithm," in Proc. of the Annual Allerton Conference on Communication, Control and Computing, vol. 41, 2003, pp. 303-311.

[11] J-G. Choi and S. Bahk, "Cell-Throughput Analysis of the Proportional Fair Scheduler in the Single-Cell Environment," IEEE Trans. Veh. Technol., vol. 56, no. 2, pp. 766-778, March 2007.

[12] H. A. Suraweera, J. T. Y. Ho, T. Sivahumaran and J. Armstrong, "An Approximated Gaussian Analysis and Results on the Capacity Distribution for MIMO-OFDM," in Proc. IEEE PIMRC, Melbourne, September 2005, pp. 211-215.

[13] P. J. Smith and M. Shafi, "On a Gaussian Approximation to the Capacity of Wireless MIMO Systems," in Proc. IEEE ICC, New York, April 2002, pp. 406-410.

[14] D. Avidor, S. Mukherjee, J. Ling and C. Papadias, "On Some Properties of the Proportional Fair Scheduling Policy," in Proc. IEEE PIMRC, New Jersey, September 2004, pp. 853-858. 